\title{
Fabrication and Assembly of a Novel High-Efficiency UHF RFID Tag on Flexible LCP Substrate
}

\author{
S. Basat*, S. Bhattacharya, A. Rida, S. Johnston, L. Yang, M.M. Tentzeris, J. Laskar \\ School of Electrical and Computer Engineering \\ Georgia Institute of Technology \\ Atlanta, GA 30332-0250, USA \\ *Email:gte309k@mail.gatech.edu
}

\begin{abstract}
In this paper, design, fabrication, assembly and testing of a unique high read-range high-efficiency (95\%) Radio Frequency IDentification (RFID) antenna for the $915 \mathrm{MHZ}$ UHF band are discussed. The exceptional characteristics of the RFID are investigated in terms of antenna-IC matching and radiation efficiency. The $915 \mathrm{MHz}$ passive tag is a 3 " $\mathrm{x}$ 3 " omnidirectional tag and yielded a read range of 31 feet compared to a 4" x 4" leading commercial design of 26 feet tested range in lab. This tag also possesses higher read power range $(-7 \mathrm{dBm}$ to $30 \mathrm{dBm})$ than the leading commercial design $(-5 \mathrm{dBm}$ to $30 \mathrm{dBm})$. The proposed RFID antenna was fabricated on 50 micron thick Liquid Crystal Polymer (LCP) substrate and the read range of the proposed RFID tags was experimentally verified. A large format LCP sheet $(300 \mathrm{~mm} \times 300 \mathrm{~mm})$ was used for antenna fabrication and the assembly of the IC was done using low temperature lead free solder alloys that are compatible with the heat distortion temperature of the LCP.
\end{abstract}

\section{Introduction}

The demand for flexible antennas with higher efficiency and more compact size has increased in the recent years mainly due to the requirements for a higher and higher read range performance of the increasingly used RFID tags and their almost ubiquitous presence in the industry in securityrelated applications.

The passive UHF RFID tags see the widest use in supply-chain and retail applications. One of the biggest advantages of passive UHF tags over the higher frequency tags (i.e. $2.45 \mathrm{GHz}$ RFID tags) is that they have a range, in many environments, of over ten feet (and sometimes as much as tens of feet). Additionally, RFID readers can scan hundreds of UHF tags simultaneously, whereas the lower frequency tags (VLF, LF, and HF bands) already suffering from limited read range ( $\sim 1-2$ feet), can handle about $10 \%$ of that scanning capacity with a lower data transfer rate.

The proposed $915 \mathrm{MHz}$ RFID tag employs far-field coupling of the real power contained in free-space propagating electromagnetic plane waves due to its shorter wavelength than, for example, the $13.56 \mathrm{MHz} \mathrm{HF}$ tags, where the inductive coupling of the transponder tag operates in the near-field as the wavelength is much longer. The IE3D and HFSS design tools are used to perform a system-level optimization of the tag, as well as to design and come up with certain antenna performance parameters such as directivity, radiation pattern, and efficiency.

It is well know that the market of the RFID tags is extremely cost sensitive. To address this issue, we have implemented large format fabrication in $300 \mathrm{~mm}$ x $300 \mathrm{~mm}$ size which can then be sectioned to individual components. The current materials set in this paper is a liquid crystal polymer, however, we are looking into substrates that are much lower cost.

During the past decade, significant research around the world has been devoted to the search for alternate lead free solder alloys. The goal simply is a drop-in replacement for eutectic tin/lead solder with good mechanical properties, plentiful supply, non-toxic and at comparable cost. A NCMS study completed in 1997 considered more than 75 different alloys, but found no drop-in replacement [1]. For lower temperature applications, eutectic tin/bismuth (42Sn/58Bi), near eutectic $\mathrm{Sn} / \mathrm{Bi}$ binary alloys, and $\mathrm{Sn} / \mathrm{Bi}$ alloys with copper, indium, gold, antimony, or zinc additions are possibilities. Surface mount joints with eutectic $\mathrm{Sn} / \mathrm{Bi}$ have proved to be more reliable in thermal cycling than eutectic $\mathrm{Sn} / \mathrm{Pb}$. However, interfacial strength and mechanical properties of the Sn-Bi alloys change with aging and are not well understood at present [2,3].

In this paper, the assembly process was optimized toward lead free and low temperature compositions that can be implemented to a wide variety of low cost organic substrates.

\section{RFID Antenna Design}

The RFID antenna structure is shown in Fig. 1. The single dipole antenna is comprised of a resistive shorting stub with length $j$ and width $i$, a double inductive stub, and a radiating body. The 250-bit read/write chip is mounted on the 4 ports, namely $R F 1, R F 2, V d d$, and $V s s$ at the feeding point as presented in Fig. $1 . R F 1$ and $R F 2$ ports are the RF signal terminals. $V d d$ is the open port to measure the IC bias voltage and $V s S$ is the ground port. The chip is designed to be operational with both single and dual dipole antennas. The RF signal ports $R F 1$ and $R F 2$ are needed to be shorted to deliver the information to the charge pump in the IC with the same phase. Time delay of the same signal at the two RF ports leads to loss of information. For the single dipole antenna, $R F 2$ port is grounded so that signalground (S-G) type of excitation can be created at the feeding point.

It is crucial to achieve high radiation efficiency for high read range since most commercial RFID antennas suffer from low efficiencies ( $50-60 \%)$ [4]. In order to accomplish maximum directivity and optimum radiation, the design is built to achieve half-wavelength $\left(\lambda_{\mathrm{r}} / 2 \sim 16 \mathrm{~cm}\right.$ in air @ $\left.915 \mathrm{MHz}\right)$ resonance at first. This was taken about to be the maximum length when the dipole antenna is stretched from one end to the other. The tapered design is proposed to obtain a smoother transition from the connecting $R F 1$ and $R F 2$ pads of the IC at the interface to the single dipole antenna to reduce reflections as much as possible. Another benefit from this tapering is used to maintain the high-efficiency when the antenna is embedded in a dielectric 
material such as LCP, although LCP's dielectric constant $(\sim 3)$ is close to the free space.

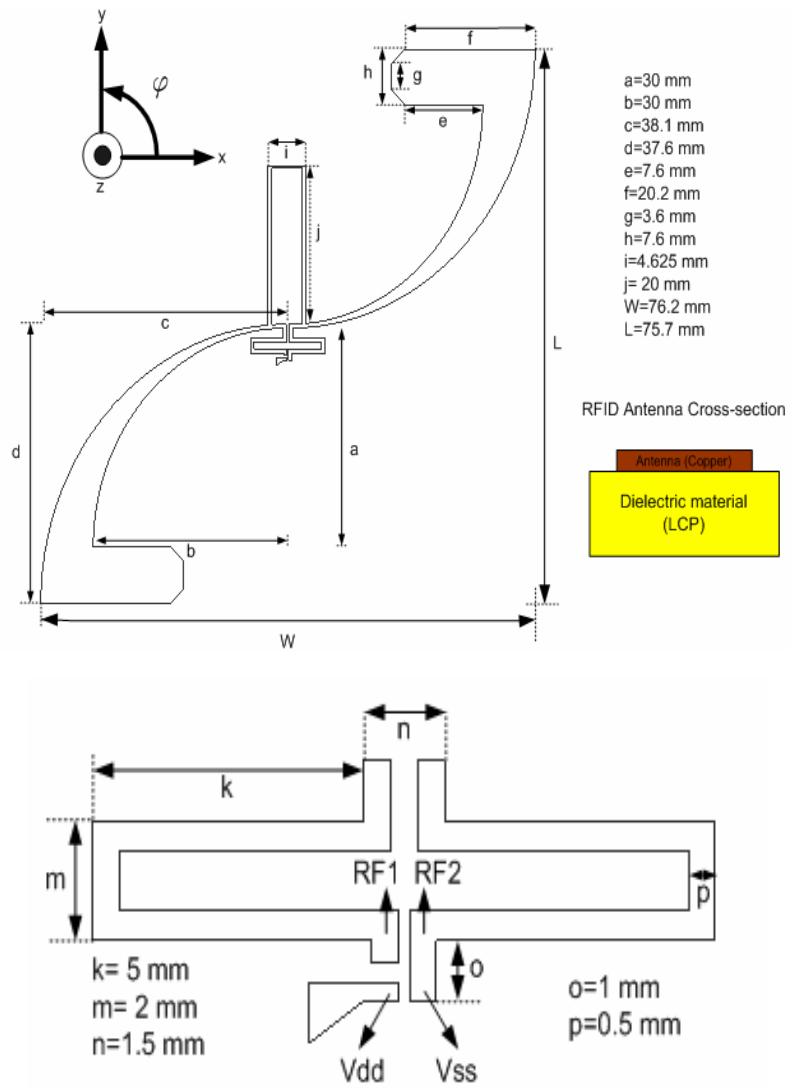

Figure 1. RFID Antenna structure and double inductive stub matching network

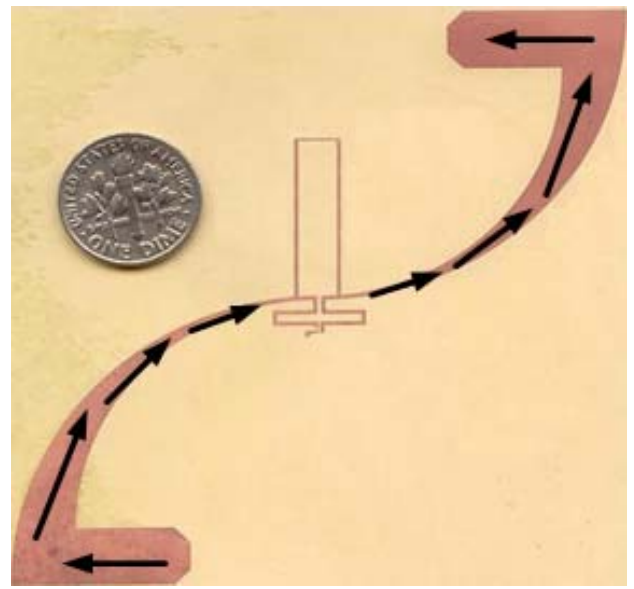

Figure 2. Fabricated RFID antenna and single dipole antenna direction of current flow

The overall matching network is designed to conjugately match a chip impedance of $73-\mathrm{j} 113$ for maximum power delivery. The resistive shorting stub and the double inductive stub make up the overall matching network to match to the chip input impedance. The shorting stub mainly controls the resistive matching and the double inductive stub controls the reactive matching. The double inductive stub structure is composed of two inductive stubs to provide symmetry on both sides of the RFID tag [5].

In Fig. 2 the fabricated 18 um thick copper antenna on flexible, low-cost, and easily manufacturable LCP $\left(\varepsilon_{\mathrm{r}}=3.16\right.$, $\tan \delta=0.002$ ) with $50 \mu \mathrm{m}$ thickness is shown. The antenna can be used for sensor applications. For this reason, the antenna is also designed to accommodate space for other surface components such as a sensor module and a battery with minimum interference to the overall antenna performance.

\section{Antenna Fabrication and RFIC Assembly}

Fabrication of the antennas was performed on 50 micron liquid crystal substrates which are bonded with 18 micron copper clad. The substrate size was $300 \mathrm{~mm}$ x $300 \mathrm{~mm}$ on which 36 antennas were fabricated in one process cycle. Commercially available 35 micron thick dry film photoresist (Riston 4615) was laminated at $160 \mathrm{~F}$ using a large area vacuum laminator. Subsequent imaging and development of photoresist and copper back etching were performed using a commercially available vertical Develop/Etch/Strip (DES) station.

The assembly challenges for the RFIDs on LCP substrates are two fold: (i) Lead Free Solder and (ii) Low Temperature Assembly. For assembly of the RFICs, low temperature solders particularly tin/bismuth alloys were employed in the initial trials in a reflow oven (BTU International, Model Pyramix 98). The temperatures and residence time in the oven were preprogrammed for the low temperature lead free assembly.

The solders were bonded to the IC using a flux and then reflowed. The alignments were inspected before and after reflow using Fein Focus X-ray machine. The IC with assembled solder balls were then placed on to the LCP substrate and passed through reflow oven again. The temperature profile for the tin/bismuth alloy was $150 \mathrm{C}$ for 60 seconds and $180 \mathrm{C}$ for 60 seconds. Finally, after reflow the interconnect integrity was examined with X-ray and DC interconnect resistance test. The known good modules were sorted for electrical as well as $\mathrm{read} / \mathrm{write}$ tests.

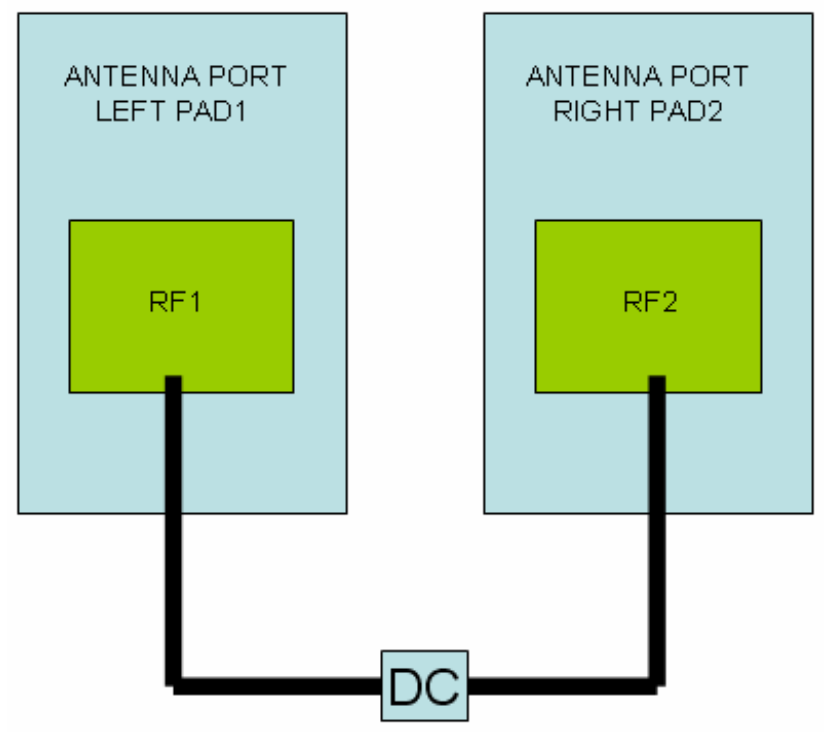

Figure 3. Antenna-IC electrical connection test setup 


\section{Electrical Characterization}

Fig. 3 shows the electrical connection test setup for the assembled ICs. A DC voltmeter with variable voltage capability was used to conduct the test. The two ports of the antenna namely $R F 1$ and $R F 2$ were connected to the meter. Since identical solders were used also on the other two ( $V d d$ and $V s s$ ) pads of the IC, good electrical connections at $R F 1$ and $R F 2$ indicate also good bonding at $V d d$ and $V s s$ pads. Intrinsically, there is a diode connecting the $R F 1$ and $R F 2$ pads in the IC. The measured characteristic curve of this diode is exhibited in Fig. 4. As it can be seen from this plot that current I is low at low level of DC supply voltage and increases with the increase in DC supply voltage. It eventually saturates to protect the IC from high current flow damage.

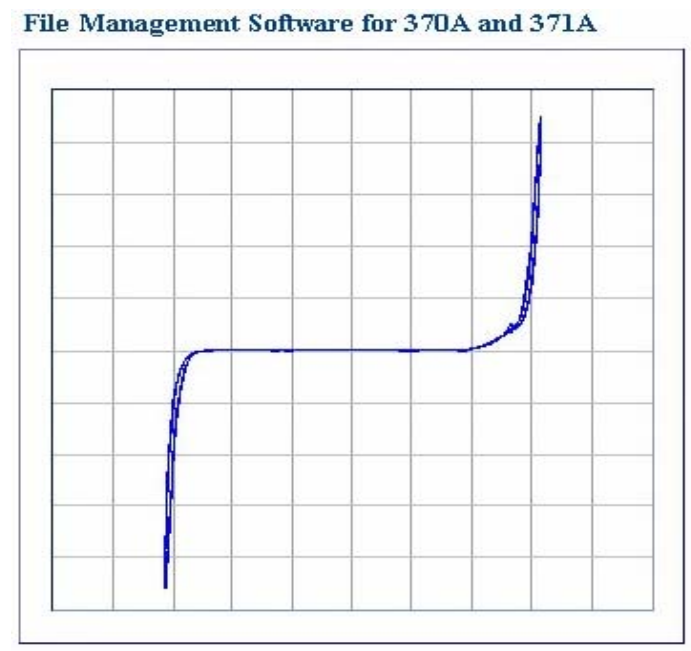

Figure 4. The I-V Curve for the diode between RF1 and RF2 pads in the IC (I- vertical and V-horizontal)

In order to measure the current I and voltage $\mathrm{V}$ levels to characterize the diode behavior, the shorting stub must be disconnected from the $R F 1$ and $R F 2$ ports of the antenna. This was achieved by merely cutting the metal line. The measured results are presented in Table 1. The packaged die results were compared to the results from the bare die on antenna. An excellent agreement between the two chips was observed as displayed in Table 1. This shows that the IC is successfully assembled.

Table 1. Packaged Die and Antenna/IC measured current and voltage values

\begin{tabular}{|c|c|c|c|}
\hline SOLDERMASK & $0.4 \mathrm{~V}$ & $0.9 \mathrm{~V}$ & $1.2 \mathrm{~V}$ \\
\hline Packaged Die & $0 \mathrm{~mA}$ & $2.20 \mathrm{~mA}$ & $43.8 \mathrm{~mA}$ \\
\hline Antenna/IC & $0 \mathrm{~mA}$ & $2.162 \mathrm{~mA}$ & $47.84 \mathrm{~mA}$ \\
\hline
\end{tabular}

Table 2. RFID antenna performance parameters and measured read range

\begin{tabular}{|c|c|c|c|c|}
\hline $\begin{array}{c}\text { Input } \\
\text { Impedance } \\
\text { (Simulate) }\end{array}$ & $\begin{array}{c}\text { Input } \\
\text { Impedance } \\
\text { (Measured) }\end{array}$ & Dir. & Eff. & $\begin{array}{c}\text { Measured } \\
\text { Read Range } \\
\text { in Lab }\end{array}$ \\
\hline $59.7+\mathrm{j} 96.4 \Omega$ & $49+\mathrm{j} 106 \Omega$ & $\begin{array}{c}2.18 \\
\mathrm{dBi}\end{array}$ & $95 \%$ & $\begin{array}{c}31 \mathrm{feet} \\
(9.45 \mathrm{~m})\end{array}$ \\
\hline
\end{tabular}

\section{Antenna Results and Discussion}

The RFID antenna performance parameters (Input Impedance, directivity, radiation efficiency, and read range) are displayed in Table 2 below. The calculated return loss [6] values at $915 \mathrm{MHz}$ based on the 73-j113 $\Omega$ chip impedance for the simulated and measured antennas are $-36.7 \mathrm{~dB}$ and $-31.7 \mathrm{~dB}$ respectively. One major factor for the high efficiency is because of the way the current flow is directed as presented in Fig. 2. Since the direction of current flow in the top and bottom parts of the antenna always add up constructively for far-field radiation, the radiation efficiency is maximized. The 5\% loss in efficiency is mainly due to the amount of radiation loss in the matching network. The RFID tag was also tested for read/write power levels. The read power range was from $-7 \mathrm{dBm}$ to $30 \mathrm{dBm}$ and the pattern generator was able to write 250 -bit user data to the memory of the chip for power levels above $2 \mathrm{dBm}$. The length $j$ of the antenna's shorting stub was reduced to half of the original length to observe the performance difference. The simulated input impedance of the antenna becomes $44+\mathrm{j} 100.1 \Omega$. The resistance drops dramatically (higher return loss); meanwhile, the inductance stays almost the same as expected. When this tag was tested, power levels were from -5 to $29 \mathrm{dBm}$ for reading and $3 \mathrm{dBm}$ for writing. The read range was measured to be close to 30 feet $(9.14 \mathrm{~m})$. This shows the effect of power transmission loss between the antenna and the matching network. More power is needed to write on the chip because of this loss in the matching network. Although the efficiency stays the same (95\%) compared to the original antenna in Table 1, read range is decreased due to the lower real part of the radiated power. The input impedance resistance goes down from $59.7 \Omega$ to $44 \Omega$ which translates as lower resistive power transfer at the antenna $+\mathrm{IC}$ interface.

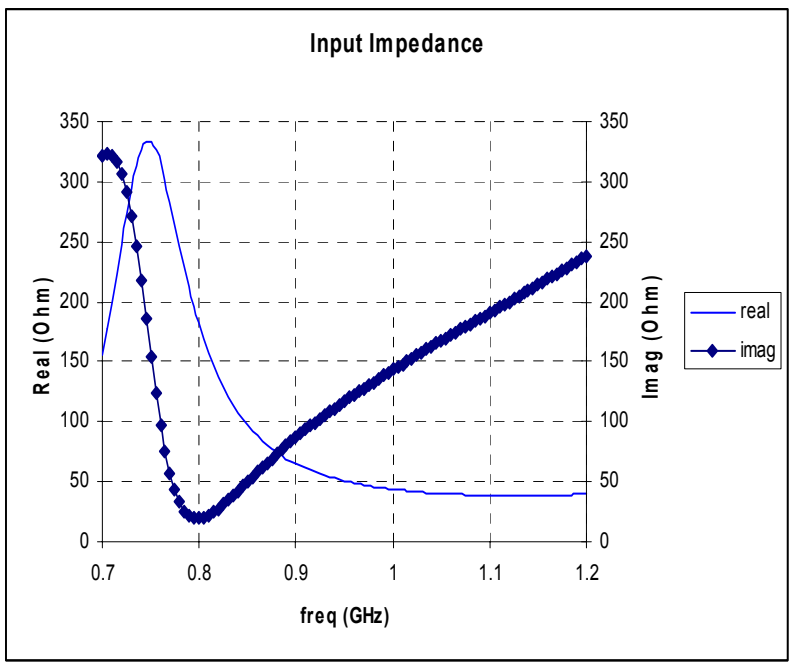

Figure 5. Input impedance of the simulated RFID antenna

The input impedance of the simulated antenna design is shown in Fig. 5. As it can be observed from the plot, the phase angle between resistance and inductance of the antenna input impedance $\left(Z_{\text {ant }}=59.7+\mathrm{j} 96.4\right)$ is lower around $915 \mathrm{MHz}$ compared to inductively coupled feed [6] matching networks. Antennas designed using inductively coupled feed structures yield high phase angles (i.e. $Z_{\text {ant }}=6.2+j 127$ ). As explained in 
the paper [7], at resonance the resistive part of the input impedance of the antenna $R_{a}$ depends only on the mutual coupling $M$; meanwhile, the inductance $X_{a}$ is dependent on the inductive loop inductance $L_{\text {loop }} . R_{a}$ is actually not only dependent on the $M$ but also on the antenna resistance $R_{r b}$. High $R_{r b}$ causes low input impedance resistance. Since the antenna shape defines $R_{r b}$, low $R_{r b}$ might not be achieved with some designs such as the one presented in [7]. For this reason, a matching network that is composed of a shorting stub to control input impedance resistance $R_{a}$ and a double inductive stub to control inductance $X_{a}$ is proposed in this paper.

In Fig. 6 the 2-D and 3-D radiation plots are shown. The 3-D radiation pattern of the antenna is doughnutshaped as expected for the general radiation pattern for a half-wavelength dipole. The 2-D polar plot shows the radiation in the two different planar cuts for the $x-z$ plane $(\varphi=0 \mathrm{deg})$ and the $y-z$ plane $(\varphi=90 \mathrm{deg})$ with angle $\theta$ that varies from 0 to 360 degrees. The pattern is almost omnidirectional with two nulls in the whole 360 degree coverage area that add up to be less than 15 degrees. This pattern mimics the radiation pattern of a half-wavelength dipole antenna as it is a tapered dipole antenna. The nulls lay horizontal to the $\mathrm{x}-\mathrm{y}$ plane where the RFID tag is least expected to transmit/receive information from the reader.
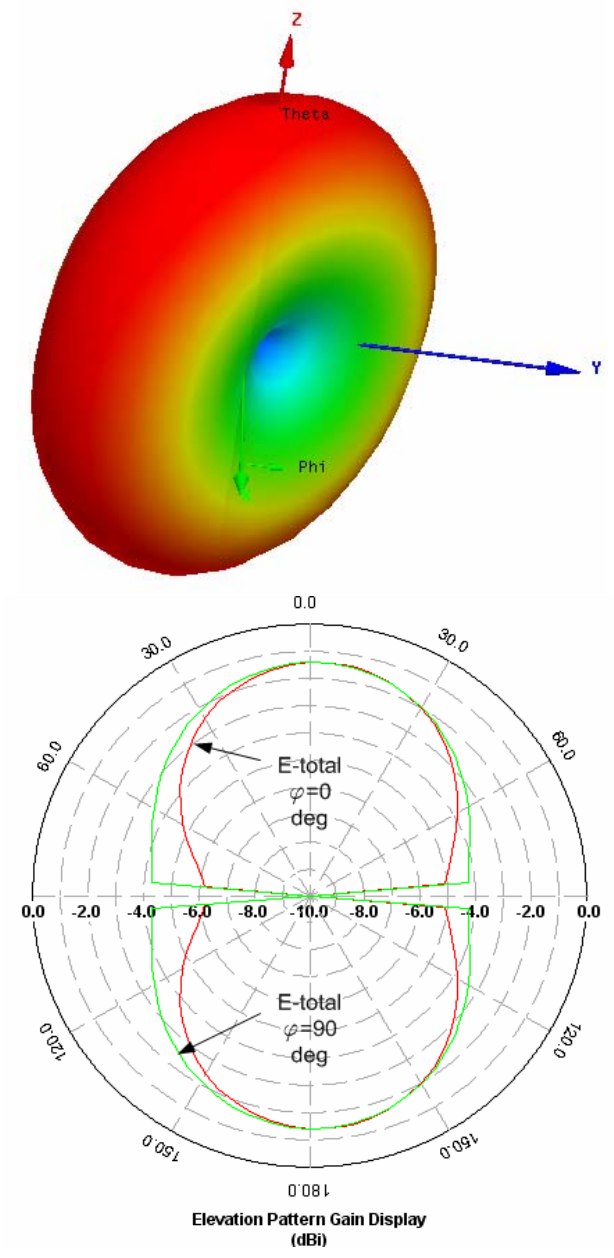

Figure 6. 3-D and 2-D far-field radiation plots

\section{Conclusions}

Maximum read range can be achieved when the dipole RFID antenna is half-wavelength resonant and has direction of current flow that adds up constructively. The tag size also plays a major role in determining the read range: The larger the tag, the larger the energy capture area, therefore the longer the read range. One major difficulty in RFID tag design is designing the matching network since the chips come with either high or low input impedance phase angles. (i.e. Philips EPC 1.19 ASIC Zc=16j350, NSC MM9647 Zc=73-j113). These three factors must be taken into account to design optimum performing RFID tags. The antennas can be fabricated using large area commercial copper etch processes. The assembly of the antennas has been optimized at lower temperatures using tin-bismuth alloys to prevent distortion of the substrate and protect the RFIC from exposure to higher temperatures.

\section{Acknowledgments}

The authors would like to acknowledge the support of the Georgia Electronic Design Center (GEDC), the NSF Packaging Research Center of Georgia Tech and the NSF CAREER Award.

\section{References}

1. J. Raby and W. Johnson, "Is lead free a wishful thinking, Semiconductor International”, August, 1999.

2. C. Melton, A. Skipor, W. Beckenbaugh, "Low temperature wetting of tin-based solder pastes", US Patent 5229070

3. C. Raeder, L. Felton, D. Knorr, G. Schmeelk, D. Lee, "Microstructural evolution and mechanical properties of SnBi based solders", Electronic Manufacturing Technology Symposium, 1993, Fifteenth IEEE/CHMT International, 4-6 Oct. 1993 Page(s):119 - 127

4. C. Cho, Hosung Choo, and Ikmo Park, "Design of UHF Small Passive Tag Antennas," IEEE Antennas and Propag. Society Inter. Symp., 2005, vol 2B, pp. 349-352

5. David M. Pozar, "Microwave Engineering Third Edition," John Wiley and Sons Inc., 2005, pp.228-235

6. P.V. Nikitin, K.V.S. Rao, S.F. Lam, V Pillai, R. Martinez, and H. Heinrich, "Power Reflection Coefficient Analysis for Complex Impedances in RFID Tag Design," IEEE Trans . Microw. Theory Tech ., 2005, vol. 53, issue 9, pp. 27212725

7. H.-W. Son and C.-S. Pyo, "Design of RFID tag antennas using an inductively coupled feed," Electronic Letters, 2005, vol. 41, no. 18, pp. $994-996$ 